\title{
Model and Algorithm for Substantiating Solutions for Organization of High-Rise Construction Project
}

\author{
Vladimir Anisimov ${ }^{1, *}$, Evgeniy Anisimov ${ }^{2}$ and Anatoliy Chernysh ${ }^{1}$ \\ ${ }^{1}$ Peter the Great St. Petersburg polytechnic University, Institute of Industrial Economics, Management \\ and Trade, 195251 St. Petersburg, Russia \\ ${ }^{2}$ Peoples' friendship University of Russia, 117198, Moscow, Russia
}

\begin{abstract}
In the paper the models and the algorithm for the optimal plan formation for the organization of the material and logistical processes of the high-rise construction project and their financial support are developed. The model is based on the representation of the optimization procedure in the form of a non-linear problem of discrete programming, which consists in minimizing the execution time of a set of interrelated works by a limited number of partially interchangeable performers while limiting the total cost of performing the work. The proposed model and algorithm are the basis for creating specific organization management methodologies for the high-rise construction project.
\end{abstract}

\section{Introduction}

Quality management projects for the construction, modernization, technical re-equipment, reconstruction or major repair of buildings and structures for various purposes, as well as their complexes is inextricably linked with the adoption of sound management decisions on the organization of material technological and logistics processes of the construction project and their financial support. Complexity of projects and significant costs, caused by possible miscalculations in the organization of these processes, lead to the fact that the relevant decisions should be based not only on experience and intuition, but also on objective scientific justifications. The tool for such justifications can be the corresponding mathematical models and algorithms [1-4].

A wide class of problems of justifying decisions on the organization of material technological and logistical processes of a construction project and their financial support can be formalized in the form of a model and algorithm for minimizing the execution time of a complex of interrelated works, with restrictions on the number of performers, their interchangeability and the cost of attracting certain work [5]. The development of such an algorithm is the purpose of this article.

\footnotetext{
* Corresponding author: an-33@yandex.ru
} 


\section{Model Description}

When building a model, the composition and interrelation of the works reflecting the corresponding material technological and logistical processes of the construction project should be displayed in the form of a network [6-8].

$$
G=\{(i, j)\}, \quad i, j=0,1, \ldots, m, \quad i<j,
$$

Authors should use the forms shown in Table 3 in the final reference list. where $i, j$ are network node numbers;

$(m+1)$ are total number of nodes.

Each task in the network (1) is associated with an arc $(i, j)$, connecting the $i$ and $j$ nodes. The node $i=0$ corresponds to the event of the beginning of the execution of the tasks package represented by the network (1). Nodes $i=1,2, \ldots, m$ the nodes correspond to the events consisting in the completion of all the tasks described by the arcs included in each of them. The total number of tasks ( $\operatorname{arcs})$ is $N$.

The sequence of task obeys the logistic rule: the task corresponding to the arc emerging from any node can not be started until all tasks corresponding to the arcs in this node is completed.

Each task $(i, j)$ is characterized by the necessary quantity $n(i, j)$ of handlers and duration $\tau(i, j)$.

A lot of handlers involved in the implementation of the set of tasks will be designated.

$$
R=\{1,2, \ldots, k, \ldots, K\}
$$

( $k$ - conditional numerical order (identifier) of handler, $K$ - number of handlers).

The interchangeability of handlers in a formalized form is represented by a matrix

Where

$$
\Delta=\left\|\delta_{k}(i, j)\right\|, k=1,2, \ldots, K, \quad(i, j) \in G,
$$

$\delta_{k}(i, j)=\left\{\begin{array}{c}1, \text { if the performer can be involved } \\ \text { to the carrying out }(i, j) \text { task, } \\ 0 \text { otherwise. }\end{array}\right.$

The cost attracts handlers to the task execution as a component of the vector

$$
\mathrm{C}=\left\|\mathrm{c}_{k}(i, j)\right\|, k=1,2, \ldots, K,(i, j) \in G,
$$

where $\mathrm{c}_{k}(i, j)$ is a cost per time unit of engaging $\mathrm{k}$ handler to execution of $(i, j)$ task.

The logistic plan for the implementation of the set of tasks is determined by the set of

$$
Y=\left\{x_{Y}(i, j), r_{Y}(i, j)(i, j) \in G, r_{Y}(i, j) \subset R\right\},
$$

where $x_{Y}(i, j)$ determined by the plan Y timestamp corresponding to the beginning of $(i, j)_{\text {task. }}$ 
$r_{Y}(i, j)$ is variety of handlers, involved to $(i, j)$ task execution in accordance with plan Y.

We will assume that the interruption of each started $(i, j) \in G$ task is not allowed and the composition of the marked handlers is not changed.

Taking into account the accepted designations, the cost $\Omega_{Y}(i, j)$ of the task execution during the $\mathrm{Y}$ plan realization is determined by the relation

$$
\Omega_{Y}(i, j)=\sum_{k \in r_{Y}(i, j)} c_{k}(i, j) \tau(i, j)
$$

Let us denote by the $G_{L}$ the set of all paths of the network (1) connecting its initial and final vertices.

The time for executing the entire set of tasks for the implementation of the plan (6) will be equal to the maximum $L \in G_{L}$ path $T_{L}$ length from the initial vertex $i=0$ of the network (1) to the final one $j=m$.

Taking into account the accepted designations, the task of forming a logistic plan (6) that minimizes the time of execution of a set of tasks (1) with restrictions on the number, interchangeability and cost of handlers in a formalized form is reduced to the following problem of mathematical programming:

define a logistics plan

$$
Y^{*}=\left\{x_{Y}^{*}(i, j), r_{Y}^{*}(i, j) \mid(i, j) \in G, r_{Y}(i, j) \subseteq R\right\}
$$

of the executing the set of tasks, ensuring compliance with the condition

$$
T^{*}=T\left(Y^{*}\right)=\min _{Y} \max _{L \in G_{L}} T_{L}(Y)
$$

under constraints

$$
\begin{gathered}
X_{Y}(i, j) \geq \max _{l, i}\left\{x_{Y}(l, i)+\tau(l, i)\right\}, \quad(i, j) \in G \\
\sum_{(i, j) \in r_{Y}(i, j)} \delta_{k}(i, j)=n(i, j) \\
\sum_{(i, j) \in F_{Y}(t)} n(i, j) \leq K \\
\sum_{k=1}^{K} \delta_{k}(i, j) \geq n(i, j) \\
\sum_{(i, j) \in G} \Omega_{Y}(i, j) \leq \Omega^{*}
\end{gathered}
$$

where $F_{Y}(t)$ is variety of the complex`s tasks, performed at each timestamp t when implementing the logistics plan $\mathrm{Y}$;

$\Omega *$ is the maximum permissible cost of the completing set of (1) tasks.

In the task (8) - (14) condition (9) formalizes the desire to minimize the time of the set of tasks execution.

Restriction (10) reflects the logistic rule that task originating from any network node (1) can begin only after the completion of all task included in this node.

Restriction (11) formalizes the requirement to allocate for each task a specified number of handlers.

Restriction (12) reflects the natural condition that the number of handlers simultaneously attracted to the task execution can not exceed their total number. 
Restriction (13) formalizes the requirement that the number and interchangeability of handlers must ensure the execution of each complex`s task (1).

Restriction (14) means that the total cost of performing the set of tasks (1) can not exceed the established permissible level.

\section{Algorithm Description}

Tasks (8) - (14) refers to nonlinear problems of discrete inhomogeneous resources distribution of an arbitrary network. It is an NP- complex object of discrete programming [9]. Precise methods for solving problems of this class were first proposed in [2]. However, in the models considered in these studies only the handlers and their productivity were taken into account. At the same time, when creating plans for realizing real material technological and logistical processes of construction production, along with renewable resources (executors), it is necessary to take into account also available non-renewable (for example, financial) resources. In tasks (8) - (14), such resources are the cost $\Omega$ Y (i,j) of performing each task when the plan is implemented. The limitations of these resources formally represents the ratio (14). The model (8) - (14) obtained as a result of introducing this relation is a further generalization of the model considered in [2] model. Exact algorithms for solving the problem (8) - (14) are currently absent. At the same time, the need for management practice for the implementation of complex projects requires their development[10]. One of the purposes of this scientific article is the construction of such an algorithm. For the existence of solution of tasks (8) - (14) it is necessary and sufficient that:

the composition and interchangeability of the handlers ensured the possibility of executing the entire set of tasks (1);

the established level $\Omega *$ of the allowable cost made it possible to carry out a set of tasks (1).

In the formalized form, the fulfillment of the first of these requirements consists in the fulfillment of the constraint (12). It means that from the staffing you can select specialists capable of performing any complex`s task (1).

In order to verify the second requirement is satisfied, can be used the set

$$
R^{*}=\left\{r^{q}(i, j) \mid(i, j) \in G, q=1,2, \ldots\right\}
$$

of all possible options for assigning resources to the relevant tasks. There are many associated with this set

$$
\Omega=\left\{\Omega^{q}(i, j), q=1,2, \ldots,(i, j) \in G\right\}
$$

of values $\Omega \mathrm{q}(\mathrm{i}, \mathrm{j})$ of the tasks execution corresponding tasks is associated with the corresponding options of resource assignment. Elements of the set (15) are defined by

$$
\Omega^{q}(i, j)=\tau(i, j) \sum_{k \in r} q_{(i, j)} c_{k}(i, j) .
$$

In view of (16), the second of the requirements ensuring the existence of a solution of the task under consideration (7) - (13), in a formalized form, is represented by the relation

$$
\sum_{(i, j) \in G} \min _{q} \Omega^{q}(i, j) \leq \Omega^{*}, q=1,2, \ldots .
$$

It is expedient to carry out a verification of the feasibility of conditions (13) and (18) before the procedure for finding the solution to problem (8) - (14) is initiated. If they are not satisfied, then the solution of problem (8) - (14) does not exist. If they are carried out, the procedure for optimizing the logistic plan for performing the set of tasks (1) can be implemented [11]. 
The basis of this procedure (the algorithm for the formation of an optimal plan) for searching can be based on the approach first proposed in [2].

It is based on the following constructions [2]:

representation of a set of $V=\{S\}$ admissible by restrictions of fragments $S$ of the logistics plan $\mathrm{Y}$ in the form of a tree of subsets (threadinging);

calculation of the lower boundary of the objective function (8) for the threads of the tree (selected subsets);

searching for feasible options for the logistics plan;

checking the established permissible options for optimality.

The procedure proposed in [2] allows to define the logistics plan (8) for the implementation of a set of tasks (1) that satisfies conditions (10) - (13).

Distinctive feature of this algorithm considered in this paper is the necessity to account, on every stage, for branching and the condition (14). If condition is broken, the execution of current thread is no longer possible and we proceed to the next thread [12].

As in the algorithm [2], branching in the proposed algorithm is carried out on the basis of the dichotomous scheme. When implemented, each vertex vs of the S-th thread of the variational tree is an element of the logistics plan. Moreover, if the task $(\mathrm{i}, \mathrm{j})$ corresponding to this element starts at the moment of time $x_{S}(i, j)$ for $r_{S}(i, j)$-th variant of handler assignment, then let us set

$$
v_{S}=\left\{x_{S}(i, j), r_{S}(i, j)\right\}
$$

If task $(\mathrm{i}, \mathrm{j})$ does not begin at the time $x_{S}(i, j)$ for $r_{S}(i, j)$-th variant of handler assignment, then let us set

$$
v_{S}=0
$$

For each thread $S \in V$ the values $x_{S}(i, j), \quad(i, j) \in G$, (initialization timestaps) must be selected from ascending sequence that corresponds to such thread $t_{S}=\left\{t_{S}^{n}\right\}, \quad n=1,2, \ldots$.

Herewith $t_{S}^{1}=0$, and the subsequent moments $t_{S}^{n}, \quad n=2,3, \ldots$ are determined on the basis of relation

$$
t_{S}^{n}=\min _{(i, j) \in F\left(t_{S}^{n-1}\right)}\left\{x_{s}(i, j)+\tau(i, j)\right\}
$$

where $F_{S}\left(t_{S}^{n-1}\right)$ is variety of tasks $(i, j)$, previously included in the S-th thread and unfinished by the time point $t_{S}^{n-1}$, that is

$$
F_{S}\left(t_{S}^{n-1}\right)=\left\{(i, j) \mid(i, j) \in G, x_{S}(i, j) \leq t_{S}^{n-1} \leq x_{S}(i, j)+\tau(i, j)\right\}
$$

Thus, it is a sequence of moments of time, in which task is completed, included in the next thread of the tree and releasing the respective handlers.

The condition $t_{S}^{1}=0$ reflects the fact that all variants of the logistics plan begin at the time $t=0$. 
Let us prove that the assignment of the dates for the commencement of task, not in accordance with the sequences $t_{s}$, does not allow to shorten the total execution time of the set of tasks under consideration (1).

Indeed, for any plan Y containing a fragment $\mathrm{S}$, the early term of the beginning of any task $(i, j) \in S$ by definition belongs to the sequence $t_{s}$. Consequently, the late dates of the beginning of the task lying on the path critical for plan $\mathrm{Y}$ also belong to this sequence. For tasks that do not belong to critical paths, it is possible to vary the timing of the start within the appropriate time reserves. At the same time, the boundaries of these reserves also belong to this sequence, and the variation within the boundaries does not change the time of execution of the set of tasks as a whole [13]. Therefore, the task starting and completion dates for the optimal logistics plan (9) according to criterion (9) must belong to the sequence corresponding to this plan $t_{s}$.

In the interest of realizing the adopted dichotomic threading scheme, we introduce the set associated with (15)

$$
D=\left\{d_{q} \mid q=1,2, \ldots\right\}
$$

in which $d_{q}=1$ if the resource assignment option $r^{q}(i, j)$ is used to execute the $(i, j)_{\text {task, or }} d_{q}=0$ otherwise.

In this case, the serial number $\mathrm{q}$ of the element $d_{q}=1$ of the set $\mathrm{D}$ characterizes both the task performed, the version of the resource assignment, and the cost of the resources involved in its implementation.

Taking into account (22), the threading process in the interests of compiling the optimal logistic plan (7) consists in choosing for each next time point $t_{S}^{n}$ the admissible variables $d_{q} \in D$ and establishing their values, that is, the relation (18) $\left(v_{S}=\left\{t_{S}^{n}, d_{q}=1\right\}\right)$ exists if the corresponding $d_{q}$ task $(i, j) \in G$ starts at the timestamp $x_{S}(i, j)=t_{S}^{n}$ with $r^{q}(i, j)$ the resource allocation option or (19) $\left(v_{S}=\left\{t_{S}^{n}, d_{q}=0\right\}\right)$, if the specified work does not begin with the considered resource assignment option at the moment $t_{S}^{n}$.

The set $P_{S}^{n}$ of variables $d_{q} \in D$ that can be included in the S-th fragment of the logistics plan (7) at the timestamp $t_{S}^{n}$, contains quantities $d_{q} \in D$, corresponding to the tasks $(i, j) \in G$ not previously included in the thread S under consideration, satisfying the conditions:

$$
\begin{gathered}
x(l, i)+\tau(l, i) \leq t_{S}^{n}, \quad(l, i) \in G \\
r^{q}(i, j) \subseteq R_{S}^{n} \\
\Omega^{*}-\sum_{m \in D_{S}\left(t_{S}^{n-1}\right)} d_{m} \Omega_{m}+\Omega_{q} \geq 0, q=1,2, \ldots,
\end{gathered}
$$


where $R_{S}^{n}$ is variety of uncommitted resources for S-th fragment of the plan at the timestamp $t_{S}^{n}$

$D_{S}\left(t_{S}^{n-1}\right)$ is a set of variables $d_{q}$ included in the thread $\mathrm{S}$ of the variants tree to the moment of time $t_{S}^{n-1}$.

Condition (24) identifies tasks for which all the preceding ones have been executed. Condition (25) identifies tasks for which there are permissible orders of free handlers, and (26) are the cost-admissible orders of free handlers.

As an estimate of the lower bound of the objective function (9), for each fragment of the calendar plan, the maximum path length from the initial vertex of the graph $G$ to the final one, determined without taking into account the resource constraints (12), (14) for tasks which are not included in S., can be adopted. In this case, if at the next thread step corresponding to the moment, it is established, then the following is supposed to be defined similarly to [2]:

a) task $(l, i) \in G$ that was previously included in the S-th fragment of the plan (the task for which $x_{S}(l, i)<t_{S} n$, begins at the appropriate moments $x_{S}(l, i)$ and ends in the moments $x_{S}(l, i)+\tau(l, i)$;

b) for the task $(i, j) \in G$ corresponding to the variable $d_{q}=P_{S}^{n}$ and, therefore, included in the considered step in the S-th thread of the tree version - $x_{S}(i, j)=t_{S}^{n}$;

в) for the task $(e, h) \in G$, corresponding to the variables $d_{u} \in P_{S}^{n}, \quad u \neq q$, which according to the resource constraint (2.9) at the moment $t_{S}^{n}$ cannot be included in the plan at the same time as the $(i, j)$, start time is $t_{S}^{n+1}$, and the duration is determined by the relation $t_{S}^{n+1}+\tau(e, h)$

If the thread is set at the considered step $d_{q}=0$, then to determine $W_{S}\left(d_{q}=0\right)$ in addition it's important following:

a) tasks $(l, i) \in G$, previously included in the S-th fragment of the logistics plan (the task for which $\left.x_{S}(l, i)<t_{S}^{n}\right)$, begin at the appropriate moments $x_{S}(l, i)$ and end in moments $x_{S}(l, i)+\tau(l, i)$;

б) the task $(i, j)$, corresponding to the variable $d_{q} \in P_{S}^{n}$, begins at the appropriate moments $t_{S}^{n+1}$ and ends in the moments $t_{S}^{n+1}+\tau(l, i)$

в) other tasks $(e, h) \in G$, corresponding to variables $d_{u} \in P_{S}^{n}, \quad u \neq q$, begin in the moment $t_{S}^{n}$ and end in the moment $t_{S}^{n}+\tau(e, h)$.

An important element of the algorithm for solving the problem (7) - (13), which significantly affects its convergence, is the method of choosing the next task and the option 
of assigning resources to it. Formally, it consists in the choice of variables $d_{q} \in P_{s}^{n}$ to include the S-th thread at $t_{S}^{n}$ time. In the proposed algorithm $d_{q}$, the selection at the next thread step is carried out in two stages: the first one selects task, and the second - the resource assignment option. The selection of the next task is carried out in accordance with the following sequence of preferences:

$$
\min T_{j}^{(n)} \rightarrow \max \tau(i, j) \rightarrow \min i \rightarrow \min j
$$

i.e., the first in the plan includes task, which corresponds to a smaller late completion date $T_{j}^{(n)}$

$j$. If there are several such tasks, then the maximum volume is selected from them. If there are several such tasks, then tasks with the smallest numbers $(i, j)$. In this case, late completion dates should be determined taking into account the considered fragment of the $\mathrm{S}$ logistic plan.

Scenario $r(i, j)_{\text {to identify the handler for the selected task }}(i, j)_{\text {is determined from the }}$ condition of the minimum value

$$
\sum_{k \in r(i, j)} \sum_{(l, h) \notin S} \delta_{k}(l, h),
$$

i.e. the handlers who are least universal for the remaining tasks $(l, h) \notin S$ are appointed The task selected in this way $(i, j)$ and scenario $r(i, j)$ the assignments of the handlers uniquely determine the next variable $d_{q} \in P_{S}^{n}$, included in S-th thread of the tree of the plan's variants at the moment $t_{S}^{n}$.

The skip of the tree is organized in accordance to the rule "go to the right". That allows storing in the memory of a computer only the current fragment of the logistics plan, the smallest of the previously obtained values of the objective function and the corresponding acceptable version of the plan.

This rule, in combination with the method of selecting tasks and handlers, is an approximate algorithm for solving the problem (8) - (14), which allows obtaining the first feasible solution in a finite number of steps equal to the number of $\mathrm{N}$ tasks in the network (1).

Each $\mathrm{S}$ thread ends if it includes all $\mathrm{N}$ tasks, that is, an acceptable logistic plan $\mathrm{Y}$ has been received, or if

$$
W_{s} \geq T^{0}(1-\mu), \quad 0 \leq \mu \leq 1,
$$

where $T^{0}$ - the least value of the objective function for the previously obtained allowable logistic plans (record);

$\mu$ - specified deviation of the objective function from the optimal (optimization accuracy).

The executive of condition (27) means that on the thread under consideration it is impossible to improve the previously obtained record by more than $100 \mu \%$ and its continuation within the specified optimization accuracy does not make sense.

The procedure for finding the solution ends if all the remaining threads satisfy the condition (27). When the method for traversing the variants tree of such a situation is established, the second return to the root vertex corresponds. At the same time, the last record is the desired value of the objective function (8), and the corresponding feasible plan for 
performing the set of operations is the optimal logistic plan[14-15]. The use of the considered algorithm provides analysis of all possible variants of the plan and excludes repetitions when viewing them.

\section{Conclusion}

In general, the proposed algorithm for solving the problem of forming an optimal plan for the implementation of a set of interrelated works provides the possibility of obtaining both exact and approximate solutions. They can be relatively simply integrated into specific decision support systems, since the requirements for the efficiency indicator and the limitations of the model (8) - (14) are sufficiently general that allows them to form a wide range of specific methodologies for justifying managerial decisions on the organization of material technological and logistical processes of the construction project and their financial support.

\section{References}

1. V.G. Anisimov, YE.G. Anisimov, A branch-and-bound algorithm for one class of scheduling problem, The magazine of computational mathematics and mathematical physics, 32 - 2, 2000-2005, (1992)

2. V.G., Anisimov, E.G. Anisimov, The magazine of computational mathematics and mathematical physics, 37-1, 54-60, (1997)

3. V.G. Anisimov, E.G. Anisimov, T.N. Saurenko, M.A. Sonkin, , Journal of Physics: Conference Series, 803, 1, 012006, (2017)

4. V. Anisimov, E. Anisimov, M.A. Sonkin, International Journal of Applied Engineering Research, 10(17), 38127-32, (2015)

5. A.R. Bril, O.V. Kalinina, I.V. Ilin, A.S. Dubgorn, O.Y. Iliashenko, Forecasting the turnover growth in the risk management system as management decisions support, Proc. of 2017 20th IEEE Inter. Conf. on Soft Computing and Measurements, SCM 2017, 7970692, 692-693, (2017)

6. A. Kozlov, S. Gutman, I. Zaychenko, E. Rytova, The formation of regional strategy of innovation-industrial development. Advances in Intelligent Systems and Computing, 37th Int. Conf. Information Systems Architecture and Technology, 15-126 (2017)

7. A. V. Izotov, O. V. Rostova, Development of a system of sectoral investment priorities, Proceedings of the 29th International Business Information Management Association Conference - Sustainable Economic Growth, Education Excellence, and Innovation Management through Vision 2020, pp. 1812-1822, (2017)

8. A.R. Bril, O.V. Kalinina, I.V. Ilin Small innovative company's valuation within venture capital financing of projects in the construction industry, MATEC Web of Conferences, 106, 08010, (2017)

9. A. O. Alekseyev, O. G. Alekseyev, V. G. Anisimov, Ye. G. Anisimov, N. I. Yachkula, Application of Markov chains in estimating the computational complexity of the simplex method, Soviet journal of computer and systems sciences, 5, 130-134, (1988)

10. O.Yu. Iliashenko, A.I. Levina, A.D. Borremans, The Approach to the Formal Specification of Static Structure of the System: mapping UML to EXPRESS-G, Proc. of the 29th IBIMA Conference - Sustainable Economic Growth, Education Excellence, and Innovation Management through Vision 2020, 1177-1186 (2017) 
11. G.Y. Silkina, Information and Communication Technologies in Ensuring of Innovative Development, Proc. of the 29th IBIMA Conf. - Sustainable Economic Growth, Education Excellence, and Innovation Management through Vision 2020, 1165-1176, (2017)

12. I.V. Ilin, O.V. Kalinina, A.I. Levina, O.Yu. Iliashenko, Approach to Organizational Structure Modelling in Construction Companies, MATEC Web of Conferences, 05028, (2016)

13. I.V. Ilin, A.I. Levina, O.Yu. Iliashenko, Enterprise architecture approach to mining companies engineering, MATEC Web of Conferences, 106, 0806 (2017)

14. A.B. Anisiforov, A.S. Dubgorn, Proc. of the 29th IBIMA Conf. - Education Excellence and Innovation Management through Vision 2020: From Regional Development Sustainability to Global Economic Growth, 2920-2930, (2017)

15. B. Ozorhon, K. Oral, Drivers of Innovation in Construction Projects, Journal of Construction Engineering and Management, 143(4), 04016118, (2017) 